\title{
Electro-optical modulation for a boron nitride nanotube probed by first-principles calculations
}

\author{
Chun-Wei Chen ${ }^{\text {a) }}$ \\ Department of Materials Science and Engineering, National Taiwan University, Taipei, Taiwan 106 \\ Ming-Hsien Lee \\ Department of Physics, Tamkang University, Tamsui, Taipei, Taiwan 251 \\ Yu-Ting Lin \\ Department of Materials Science and Engineering, National Taiwan University, Taipei, Taiwan 106
}

(Received 13 August 2006; accepted 26 September 2006; published online 27 November 2006)

\begin{abstract}
The electro-optical modulation effect of a $(10,0)$ boron nitride nanotube is investigated by first-principles, density functional theory calculations. The optical properties of boron nitride nanotubes can be tuned through perturbation by a transverse electric field. Large optical anisotropy in variations of the absorption spectra and refractive indices in response to external electric field is found. In addition, a method called the band-resolved absorption density analysis technique is developed to investigate the detailed optical absorption transition processes. The result is consistent with the recent experimental observation [M. Ishigami et al., Phys. Rev. Lett. 94, 56804 (2005)] and suggests that the boron nitride nanotubes can be a promising material for electro-optical modulation device applications. () 2006 American Institute of Physics. [DOI: 10.1063/1.2370561]
\end{abstract}

From the time of their discoveries, ${ }^{1}$ carbon nanotubes (CNTs) have been receiving considerable interest due to their unique electronic and mechanic properties. ${ }^{2-4}$ Similar to the structure of CNTs formed by rolling a graphene sheet, the existence of boron nitride nanotubes (BN-NTs) by rolling a boron nitride sheet was predicted theoretically ${ }^{5}$ and synthesized experimentally shortly after. ${ }^{6}$ As far as the optical and optoelectronic applications of nanotubes are concerned, BNNTs could be superior to CNTs since they exhibit only semiconducting properties independent of chirality and diameter with wide band gaps ranging from 4.0 to $5.5 \mathrm{eV}{ }^{5,7}$ Previous calculations $^{8,9}$ showed that modifications in the electronic structures of CNTs and BN-NTs can be achieved by applying a transverse electric field, leading to the reduction and even complete closing in the band gap. This so-called giant Stark effect (GSE), is further confirmed experimentally by bias dependent scanning tunneling microscopy. ${ }^{10}$ Through the Stark effect, the band structures of the BN-NTs can be modified by coupling the neighboring subbands, resulting in a significant change in their electronic structures. The effect is more pronounced in BN-NTs than in CNTs due to a reduced screening of the electric field by their large band gap values. ${ }^{9}$

In this letter, we would like to employ first-principles calculations to investigate the variations of optical properties of the $(10,0)$ BN-NT tuned by a transverse electric field. Two main modifications of optical parameters perturbed by a transverse electric field will be addressed: (1) absorption changes and (2) refractive index changes. The former is usually called the electro-absorption effect and used through the modification of transmitted beams, the latter usually called the electro-optical effect and used in light-phase-sensitive situations. ${ }^{11}$ In addition, a method called the band-resolved absorption density analysis technique is developed to investigate the optical transition mechanism involved in the absorption processes under the influence of transverse electric fields.

\footnotetext{
a) Author to whom correspondence should be addressed; electronic mail: chunwei@ntu.edu.tw
}

CASTEP (Refs. 12 and 13) a plane-wave pseudopotential program based on the density functional theory (DFT) and local density approximation (LDA) is used to carry out the electronic structure calculations. To study the effect of a transverse electric field on the electronic structure of the nanotube, a uniform external electric field ( $z$ direction) is applied perpendicular to the tube axis by using a sawtoothtype potential compatible with the periodic condition. A large kinetic-energy cutoff of $700 \mathrm{eV}$ and a $k$-point sampling using a Monkhorst-Pack grid with a $0.02 \AA^{-1} k$-point spacing along the tube axis is used to ensure the convergence in the calculations. The structures of $(10,0)$ BN-NTs under different transverse electric fields are fully optimized until the force on each atom during relaxation is less than $0.03 \mathrm{eV} / \AA$. The optical properties are calculated based on the independentparticle approximation, i.e., the excitonic effects and the local-field corrections are neglected. To predict optical properties, we first calculate the imaginary part of the dielectric function $\varepsilon_{2}(\omega)$ using the usual perturbation-theory-derived sum-over-states formalism,

$$
\varepsilon_{2}(\omega)=\frac{4 \pi^{2}}{\Omega \omega^{2}} \sum_{i \in \mathrm{VB}, j \in \mathrm{CB}} \sum_{k} w_{k}\left|p_{i j}\right|^{2} \delta\left(E_{k j}-E_{k i}-\omega\right),
$$

where $\Omega$ is the unit cell volume, and $\omega$ is the photon energy. The VB and CB denote the valence and conduction bands, respectively. The $p_{i j}$ denotes the dipole transition matrix elements obtained from the self-consistent band structure calculations and $w_{k}$ is the $k$ th point weighting. The effective unit cell volume $\Omega$ of a nanotube, instead of the volume of a supercell, is used in the calculations of optical dielectric functions. The value of $\Omega$ is therefore given by $\Omega$ $=\pi(d / 2)^{2} \times a$, where $d$ and $a$ are the diameter and the length of the translational vector of a nanotube, respectively. The real part of the dielectric function $\varepsilon_{1}(\omega)$ is obtained by the Kramers-Kronig transformation. All other linear optical properties such as absorption coefficient and refractive index can be obtained as functions of both the real and imaginary parts of dielectric function. 

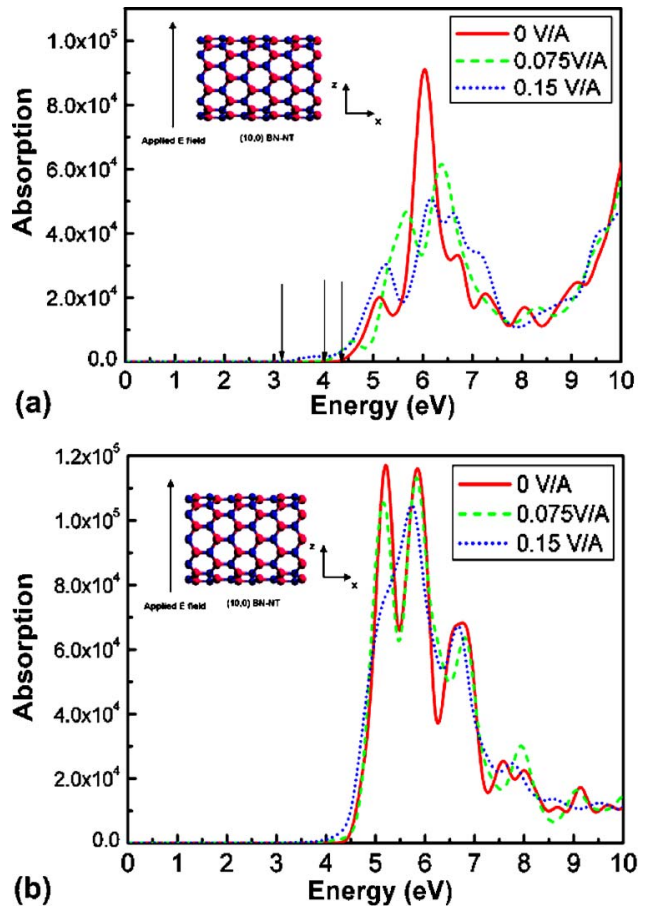

FIG. 1. (Color online) Variations of absorption spectra of $(10,0)$ BN-NT under different transverse electric fields of $0,0.075$, and $0.15 \mathrm{~V} / \AA ̊ .\left(F_{\text {ext }} \| z\right)$ (a) $\alpha_{\perp}(\omega)$ for perpendicular polarization $(E \| z)$. (b) $\alpha_{\|}(\omega)$ for parallel polarization $(E \| x)$. The arrows indicate the evolution of the band gap size with increasing electric field.

In the previous calculations, ${ }^{9}$ it was found that the band gap of the BN-NT can be tuned as the result of significant subband mixing caused by the perturbation of an external electric field perpendicular to the tube axis. The valence band edge state rises in energy with its charge density moving along the direction of the electric field and being highly localized on the $\mathrm{N}$ atoms. The conduction band edge, on the other hand, lowers its energy with its charge density moving opposite to the direction of the electric field and being highly localized on the B atoms. This accounts for the reduction in the band gap values with applied electric fields. Figure 1(a) demonstrates the evolution of absorption spectra $\alpha_{\perp}(\omega)$ of a $(10,0)$ BN-NT under different strengths of transverse electric fields along the $z$ direction $\left(F_{\text {ext }} \| z\right)$ with optical polarization perpendicular to the tube axis $(E \| z)$. The shapes and peak positions of the absorption spectrum at zero electric field $\left(F_{\text {ext }}=0 \mathrm{~V} / \AA\right)^{\prime}$ show a good agreement with recent DFT calculations. ${ }^{14}$ By increasing the electric field, the absorption edge is found to occur below the fundamental band edge at zero electric field due to the fact of the reduction in the band gap. In addition, the main absorption peaks smear out as the applied electric field is increased. The overall absorption spectra move toward lower energy positions and the oscillator strength of the main peaks gradually decreases with increasing electric field. This demonstrates significant electroabsorption properties of BN-NTs. The situation is quite different in the absorption spectra $\alpha_{\|}(\omega)$ with optical polarization along the tube axis $(E \| x)$. Figure $1(b)$ shows the evolution of absorption spectra $\alpha_{\|}(\omega)$ of the $(10,0)$ BN-NT under the same transverse electric fields. Unlike the drastic change in the absorption spectra $\alpha_{\perp}(\omega)$, the $\alpha_{\|}(\omega)$ spectra demonstrate less sensitive characters to transverse electric fields without significant smearing out features as seen in $\alpha_{\perp}(\omega)$. The above result suggests that large optical anisotropy in Downloaded 09 Dec 2008 to 140.112 .113 .225 . Redistribution subject
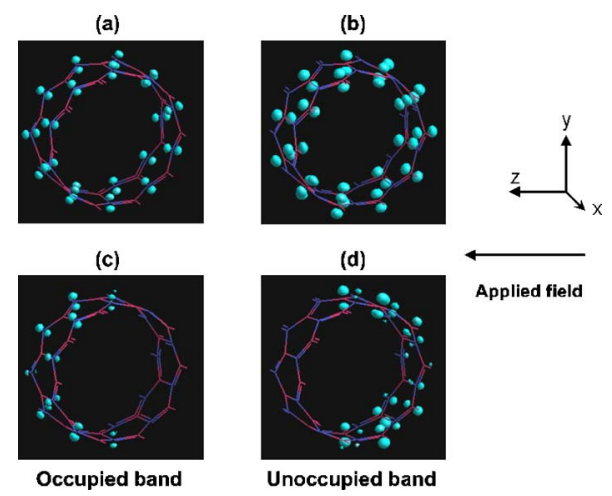

Applied field

Occupied band

Unoccupied band

FIG. 2. (Color online) Plots of absorption densities represent the transitions from (a) occupied bands and (b) unoccupied bands, corresponding to the absorption peak at $6.05 \mathrm{eV}$ in the $\alpha_{\perp}(\omega)$ under zero electric field. (c) and (d) represent the absorption densities of the occupied and unoccupied bands, respectively, corresponding to the absorption peak at $6.12 \mathrm{eV}$ in the $\alpha_{\perp}(\omega)$ under a transverse electric field of $0.15 \mathrm{~V} / \AA$. (Isosurface for these plots is equal to the 0.5 maximum value.)

electro-absorption modulation can be achieved in BN-NTs.

In order to investigate the field effect on the optical transitions in BN-NTs, the band-resolved absorption density analysis technique is applied to investigate the mechanism of optical absorption processes. We use a weighted-density analysis scheme to visualize "absorption strength" throughout the entire space. The idea is to compute effective absorption coefficients for each band through a "band-resolved" partial summing strategy, ${ }^{15}$ this gives a weighting factor when normalized by the total absorption coefficient. To perform band-resolved analysis on the absorption coefficient $\alpha(\omega)$, we rewrite it to be explicitly containing $\varepsilon_{2}(\omega)$ in the numerator, so that $\alpha(\omega)=\varepsilon_{2}(\omega) \omega / n(\omega) c$, where the refractive index $n(\omega)$ (which always has a value larger than 1) in the denominator is a slower varying function compared with $\varepsilon_{2}(\omega)$. We approximately define our band-resolved partial absorption coefficients as $\alpha_{i k}^{\text {occ }}(\omega)=\varepsilon_{2, i k}^{\text {occ }}(\omega) \omega / n(\omega) c$ for the $i$ th occupied state in $k$-point $k$, and $\alpha_{j k}^{\text {unocc }}(\omega)$ $=\varepsilon_{2, j k}^{\text {unocc }}(\omega) \omega / n(\omega) c$ for the $j$ th unoccupied state in $k$-point $k$. Under such construction, band-resolved $\alpha(\omega)$ is related to band-resolved $\varepsilon_{2}(\omega)$. The band-resolved $\varepsilon_{2, i k}^{\text {occ }}(\omega)$ and $\varepsilon_{2, j k}^{\text {unocc }}(\omega)$ can be obtained by fixing one occupied band index $i$ or one unoccupied band index $j$, respectively, and sum the rest of the band indices in the double summation as described in Eq. (1). These weighting factors for "occupied" (or "unoccupied") bands can be written as $w_{\omega, i k}^{\text {occ }}$ $=\alpha_{i k}^{\text {occ }}(\omega) / \alpha(\omega)$ and these weighting factors can then be used to construct the occupied band (valence band) absorption probability densities,

$$
\rho_{\omega}^{\mathrm{occ}}(r)=\sum_{k} \sum_{i=1}^{\mathrm{occ}} w_{\omega, i k}^{\mathrm{occ}}\left|\psi_{i k}(r)\right|^{2} w_{k},
$$

$\rho_{\omega}^{\text {occ }}(r)$ corresponds to all the contributions from occupied bands to the absorption coefficient at frequency $\omega$, where $\psi_{i k}(r)$ is the wave function in the $i$ th occupied band and in the $k$-point $k$, and $w_{k}$ is the $k$ th point weighting. The same scheme can be applied to obtain the unoccupied band (conduction band) absorption probability densities. Note that the above weighting factors, and therefore weighted densities, are frequency dependent, which means that for a given energy/frequency in absorption spectra, one can obtain the three-dimensional density distribution which represents the to AIP license or copyright; see http://apl.aip.org/apl/copyright.jsp 


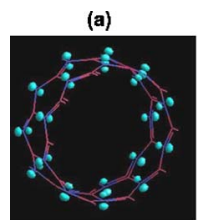

(c)

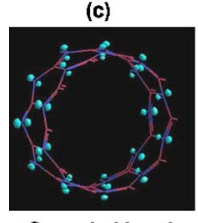

Occupied band
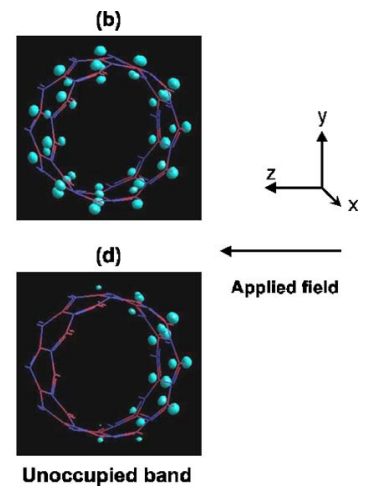

Unoccupied band
FIG. 3. (Color online) Plots of absorption densities represent the transitions from (a) occupied bands and (b) unoccupied bands, corresponding to the absorption peak at $5.83 \mathrm{eV}$ in the $\alpha_{\|}(\omega)$ under zero electric field. (c) and (d) represent the absorption densities of the occupied and unoccupied bands, respectively, corresponding to the absorption peak at $5.76 \mathrm{eV}$ in the $\alpha_{\|}(\omega)$ under a transverse electric field of $0.15 \mathrm{~V} / \AA$. (Isosurface for these plots is equal to the 0.5 maximum value.)

most important occupied (or unoccupied) electronic structure subsystems that play the major role in the optical absorption processes.

Figures 2(a) and 2(b) show the plots which denote the main contributions for the absorption peak at $6.05 \mathrm{eV}$ in the $\alpha_{\perp}(\omega)$ (perpendicular polarization) under zero electric field, by summing all the orbital densities involved in the optical transitions from the occupied and unoccupied bands, respectively. It is found that the off-site optical transitions play the dominant role in the observed absorption peak. The absorption densities are evenly distributed along the circumferential direction both in the occupied and unoccupied bands for the unperturbed BN-NT. The absorption densities in the occupied bands are located on $\mathrm{N}$ atoms and those in the unoccupied bands are located on B atoms. The more localized feature on $\mathrm{N}$ atoms (with a smaller volume of density distribution) in the occupied band reflects the characters of an ionic semiconductor of the BN-NT with tightly binding valence electrons. The $p_{z}$-like orbital shapes both in the occupied and unoccupied bands suggest that the absorption peak mainly results from $\pi \rightarrow \pi^{*}$ transitions. Figures 2(c) and 2(d) show the plots of absorption densities corresponding to the main peak at $6.12 \mathrm{eV}$ in the $\alpha_{\perp}(\omega)$ when $F$ $=0.15 \mathrm{~V} / \AA$. The absorption densities for the occupied and unoccupied bands are polarized along the opposite directions. Since the spatial overlap between the two bands is decreased, the overall transition resulting from these two bands will be weakened by electric field. This accounts for the gradual quench in the absorption peak with electric field as seen in Fig. 1(a). In figure 3 it is found that spatial overlap between the occupied and unoccupied bands from the absorption density distribution is much larger in the $\alpha_{\|}(\omega)$ than in the $\alpha_{\perp}(\omega)$. As a result, the $\alpha_{\|}(\omega)$ spectra with $(E \| x)$ demonstrate less sensitive characters with respect to the $\alpha_{\perp}(\omega)$ counterpart with $(E \| z)$ in response to the perturbation of transverse electric field.

Figures 4(a) and 4(b) show the variations of refractive indices $n(\omega)$ of the $(10,0)$ BN-NT under different electric fields. It is found that the change in the refractive index $\Delta n$ for perpendicular polarization $(E \| z)$ is more pronounced with respect to that for parallel polarization $(E \| x)$, giving the potential application in the electro-optical effect for light-
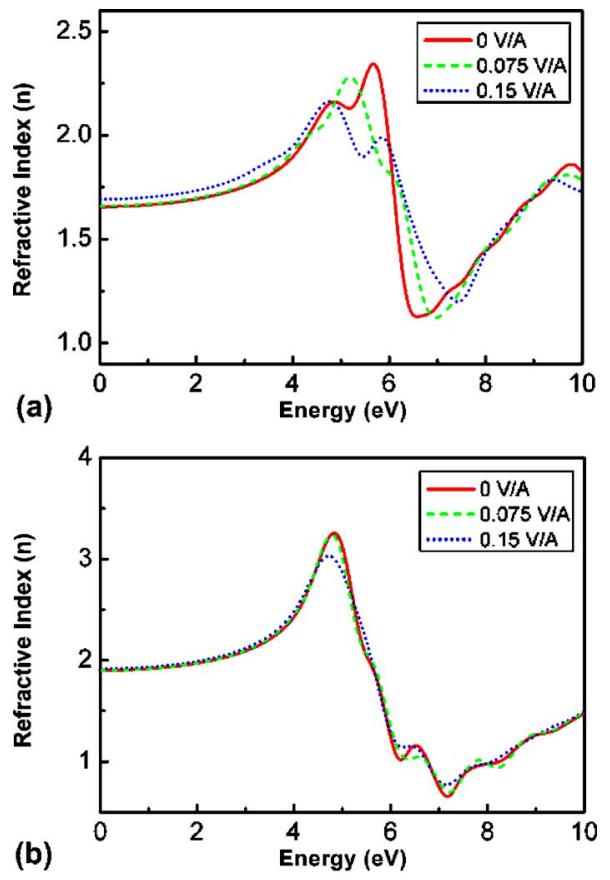

FIG. 4. (Color online) Variations of refractive indices $n(\omega)$ of the $(10,0)$ BN-NT under different transverse electric fields of $0,0.075$, and $0.15 \mathrm{~V} / \AA$. $\left(F_{\text {ext }} \| z\right)$ (a) for perpendicular polarization $(E \| z)$ and (b) for parallel polarization $(E \| x)$.

phase-sensitive nanodevices. Due to the nature of small diameter in BN-NTs, the threshold voltage required for the electro-optical effect is very small compared to that in the other bulk materials, which makes the BN-NTs a promising material for potential electro-optical modulation device applications.

This work is supported by National Science Council, Taiwan with Project Nos. of NSC94-2120-M-002-005, NSC94-2120-M-002-015, and 94-2112-M-002-040. The authors also thank the National Center for High-Performance Computing for providing CPU times.

${ }^{1}$ S. Iijima, Nature (London) 56, 345 (1991).

${ }^{2}$ R. Saito, G. Dresselhaus, and M. S. Dresselhaus, Physical Properties of Carbon Nanotubes (Imperial College Press, London, 1998).

${ }^{3}$ P. Kim and C. M. Lieber, Science 286, 2148 (1999).

${ }^{4}$ S. J. Tan, A. R. M. Verschueren, and C. Dekker, Nature (London) 393, 49 (1998).

${ }^{5}$ A. Rubio, J. L. Corkill, and M. L. Cohen, Phys. Rev. B 49, 5081 (1994); X. Blase, A. Rubio, S. G. Louie, and M. L. Cohen, Europhys. Lett. 5, 335 (1994).

${ }^{6}$ N. G. Chopra, R. J. Luyken, V. H. Crespi, M. L. Cohen, S. G. Louie, and A. Zettl, Science 269, 966 (1995).

${ }^{7}$ G. G. Fuentes, E. Borowiak-Palen, T. Pichler, X. Liu, A. Graff, G. Behr, R. J. Kalenczuk, M. Knupfer, and J. Fink, Phys. Rev. B 67, 035429 (2003).

${ }^{8}$ K. H. Khoo, M. S. C. Mazzoni, and S. G. Louie, Phys. Rev. B 69, 201401 (2004).

${ }^{9}$ C. W. Chen, M. H. Lee, and S. J. Clark, Nanotechnology 15, 1837 (2004).

${ }^{10}$ M. Ishigami, J. D. Sau, S. Aloni, M. L. Cohen, and A. Zettl, Phys. Rev. Lett. 94, 056804 (2005).

${ }^{11}$ S. Schmitt-Rink, D. S. Chemla, and D. A. B. Miller, Adv. Phys. 38, 89 (1989).

${ }^{12}$ M. C. Pyane, M. Teter, D. C. Allan, and J. D. Joannopoulos, Rev. Mod. Phys. 64, 1045 (1992).

${ }^{13}$ A. J. Read and R. J. Needs, Phys. Rev. B 44, 13071 (1991).

${ }^{14}$ G. Y. Gou and J. C. Lin, Phys. Rev. B 71, 165402 (2005).

${ }^{15}$ M. H. Lee, C. H. Yang, and J. H. Jan, Phys. Rev. B 70, 235110 (2004). 